\title{
Orchestrating Emotion and Action in an Evolutionary Framework
}

\author{
"Comment on "The Quartet Theory of Human Emotions: An Integrative and Neurofunctional Model" by \\ S. Koelsch et al. \\ Michael A. Arbib \\ ABLE Project (Action, Brain, Language, Evolution), Computer Science, and Neuroscience \\ University of Southern California \\ Los Angeles, CA 90089-2520, USA \\ arbib@usc.edu
}

The lead author of the Quartet Theory (Koelsch et al 2015) is, appropriately enough, an expert on the neuroscience linking music and emotion, and examples of this linkage are a welcome feature of the article. Actually, the article charts two quartets:

A structural quartet of affect systems centered on (i) brainstem, (ii) diencephalon, (iii) hippocampus and (iv) orbitofrontal cortex.

A functional quartet of components that contribute to emotion percepts: (1) an affective component due to projections from the affect systems to insular and somatosensory cortices; (2) a sensory-interoceptive component synthesized in the insular cortex based on the integration of the physiological, including homeostatic and vegetative, condition of the body; (3) a motor component, that is, the tendency (or drive) to act, originating from the basal-ganglia as well as from the orbitofrontal cortex; and (4) a cognitive component involving conscious cognitive appraisal.

Around half the text is devoted to a masterly summary of relevant neuroscience literature, with one section devoted to each player in the structural quartet. This review of over 200 citations alerted me to many important papers that expanded my knowledge of key findings in the neuroscience of emotion. The aim of this commentary, then, is to sound some complementary notes. Forgive me if I sound my own horn. I claim no particular expertise in the field of emotion, but several of my publications seem appropriate for this commentary.

I had the pleasure of coauthoring an article on "Primate Vocalization, Gesture, and the Evolution of Human Language" with Katja Liebal and Simone Pika (Arbib et al 2008), and had thus expected (wrongly) to see some influence of Katja's work on apes in placing emotion in an evolutionary perspective. The authors do note that the affect systems of the structural quartet "were increasingly differentiated during the course of evolution, and each of these systems generates a specific class of affects (e.g., ascending activation, pain/pleasure, attachment-related affects, and moral affects)" (from the Abstract). However, the only explicit discussion of evolution is to make a crucial distinction: "Once an organism has satisfied bodily needs and achieved homeostasis, the organism is satiated, and stimuli that were incentive before can become even aversive .... This is in contrast to the hippocampuscentered tender positive affects, which do not satiate. Note that a brain system for attachmentrelated affect that does not satiate is evolutionary adaptive, because feeling attached to a child, loving a child and feeling the joy of being together with the child are emotions that serve the continuous protection, and nurturing of the offspring" (\$2.3.3). 
I find this distinction very useful. In thinking about emotion in an evolutionary manner, I have been influenced in part by Denton et al.'s (2008) notion of "primordial emotions" as the subjective element of genetically programmed behavior patterns which support homeostasis. They include "thirst, hunger for air, hunger for food, pain and hunger for specific minerals etc. There are two constituents of a primordial emotion--the specific sensation which when severe may be imperious, and the compelling intention for gratification by a consummatory act. ... It is hypothesized that early in animal evolution complex reflex mechanisms in the basal brain subserving homeostatic responses, in concert with elements of the reticular activating system subserving arousal, melded functionally with regions embodied in the progressive rostral development of the telencephalon. This included the emergent limbic and paralimbic areas, and the insula" (from their abstract). What Koelsch et al remind us is that the ancient instincts in which one may search for the evolutionary roots of emotion include social instincts as well as the consummatory acts emphasized by Denton et al. Indeed, one may recall the 4Fs of Pribram (1960) - feed, fight, flee and reproduce - as relevant to this expansion.

Let's return to the phrase "hippocampus-centered tender positive affects." It reveals a problem that is somewhat troublesome, though it is one that I think most of us fall prey to. They say that "the anterior hippocampus is preferentially involved in emotional functions (such as music-evoked emotions and autobiographical memory) ... and the posterior hippocampus is preferentially involved in cognitive functions (such as spatial memory and navigation). ... this view emphasizes that cognitive and emotional functions are co-localized in the hippocampus" (§2.3). But having made the important distinction between anterior and posterior hippocampus the authors have convinced us that being in the hippocampus more broadly does not imply colocalization. To what extent, then, are there crucial distinctions that talk of a hippocampuscentered system obscures. The case is perhaps even more tenuous for the diencephalon-centered system, since this lumps the hypothalamus with the thalamus, and the thalamus has diverse nuclei, some more related to specific sensory systems, some linking different regions of cerebral cortex and some linking them with basal ganglia and cerebellum. This suggests that the structural quartet must be replaced, by at least, a small orchestra; and the orchestra analogy reminds us that it is a mistake to link region $\mathrm{A}$ to function $\mathrm{X}$-rather, we must seek the minimal systems that allow us to characterize key data on each function of interest.

The latter point links into my concerns as a computational neuroscientist. A neurophysiologist may observe that particular macaque neuron firing can be correlated with a specific aspect of action, perception, emotion and/or memory, etc. Similarly a neuropsychologist may link a lesion of region $A$ to a deficit in function $X$, while a brain imager may find that region $A$ is more active in task $X$ than in task $Y$, and all end up talking as if region $A$ is responsible for task $X$. By contrast, the brain modeler must seek to link region $A$ to other regions, spanning from input to output and including the updating and readout of memory states to offer explicit hypotheses on how circuitry in region A works in concert with circuitry in other brain region when task $X$ is being executed - and yet also illuminate why $A$ plays a role (albeit with less overall activity) during task $\mathrm{Y}$ as well.

Such models may implicate emotions not only in, e.g., their facial expression (something to be analyzed for its role in social competition and cooperation) but also in the sort of individual practical behavior that changes, and changes with, emotional state. I have no such models of my own of emotion processing as such, but readers may find the following topics of interest: a methodology for linking models rooted in data on macaque circuitry to explanations of human brain imaging data (Arbib et al 
2000); modeling of the interaction of hippocampus with other brain regions in spatial behavior linked to drives/primordial emotions (Arbib \& Bonaiuto 2012, Guazzelli et al 1998, Lieblich \& Arbib 1982); and a paper (Arbib \& Fellous 2004) and an edited collection of articles (Fellous \& Arbib 2005) seeking to bridge the gap between the neurobiology of emotion and the design of robots for which (something like) emotions play a role in their unfolding behavior.

Finally, I welcome $\S 4$, Interactions with the language system, and only regret its brevity. A fuller exploration of the way in which language emerges from and in turn enriches other systems is a topic to be developed elsewhere (and not necessarily by me - an old article, Arbib 2001, is at best a down payment) - as is the relation between the more emotion-linked semantics of music and the more propositional semantics of language (as discussed by Seifert et al 2013 in a chapter which appeared in a book to which Koelsch and Liebal both contributed).

I close by reiterating my admiration for the massive amount of literature reviewed by Koelsch and his colleagues. My aim has been to set forth some of the challenges of making full use of their database: developing an evolutionary framework to more fully link emotions to different patterns of social and individual behavior (including an understanding of when emotions prove maladaptive, Arbib 2005); and assessing how to refine the structural quartet into a somewhat finer subdivision into subsystems that can more adequately be integrated into computational models of the functional quartet.

Acnowledgement: This material is based in part on work supported by the National Science Foundation under Grant No. BCS-1343544 "INSPIRE Track 1: Action, Vision and Language, and their Brain Mechanisms in Evolutionary Relationship" (Michael A. Arbib, Principal Investigator)."

\section{References}

Arbib MA. 2001. Co-evolution of human consciousness and language. Ann N Y Acad Sci 929: Cajal and Consciousness: Scientific Approaches to Consciousness on the Centennial of Ramon y Cajal's Textura 195-220

Arbib MA. 2005. Beware the Passionate Robot In Who needs emotions? The brain meets the robot, ed. J-M Fellous, MA Arbib. New York: Oxford University Press

Arbib MA, Billard A, lacoboni M, Oztop E. 2000. Synthetic brain imaging: grasping, mirror neurons and imitation. Neural Networks 13: 975-97

Arbib MA, Bonaiuto JJ. 2012. Multiple Levels of Spatial Organization: World Graphs and Spatial Difference Learning. Adaptive Behavior 287-303: 287-303

Arbib MA, Fellous JM. 2004. Emotions: from brain to robot. Trends Cogn Sci 8: 554-61

Arbib MA, Liebal K, Pika S. 2008. Primate Vocalization, Gesture, and the Evolution of Human Language. Current Anthropology 49: 1053-76

Denton DA, McKinley MJ, Farrell M, Egan GF. 2008. The role of primordial emotions in the evolutionary origin of consciousness. Consciousness and cognition 18: 500-14

Fellous J-M, Arbib MA, eds. 2005. Who Needs Emotions: The Brain Meets the Robot. Oxford, New York: Oxford University Press.

Guazzelli A, Corbacho FJ, Bota M, Arbib MA. 1998. Affordances, Motivation, and the World Graph Theory. Adaptive Behavior 6: 435-71

Koelsch S, Jacobs AM, Menninghaus W, Liebal K, Klann-Delius G, et al. 2015. The Quartet Theory of Human Emotions: An Integrative and Neurofunctional Model. Physics of Life Reviews (this issue) 
Lieblich I, Arbib MA. 1982. Multiple Representations of Space Underlying Behavior. The Behavioral and Brain Sciences 5: 627-59

Pribram KH. 1960. A review of theory in physiological psychology. Annual Rev. Psychol. 11

Seifert U, Verschure PFMJ, Arbib MA, Cohen AJ, Fogassi L, et al. 2013. Semantics of Internal and External Worlds In Language, Music, and the Brain: A Mysterious Relationship, Strüngmann Forum Reports, vol. 10, ed. MA Arbib. Cambridge, MA: MIT Press 